\title{
Censos 2017: Existe Número Suficiente de Anestesiologistas em Portugal?
}

\section{Census: Are There Enough Anaesthesiologists in Portugal?}

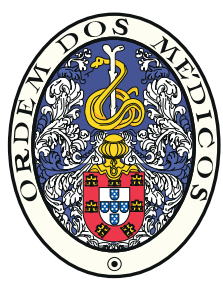

Paulo LEMOS $\bigotimes^{1}$, Alexandra GUEDES 1 , Joana MOURÃO ${ }^{1}$, Joaquim Figueiredo LIMA¹, José VEIGA¹, Manuel CHEDAS ${ }^{1}$, Pedro BRANCA ${ }^{1}$, Rosário ABRUNHOSA ${ }^{1}$, Susana CADILHA ${ }^{1}$, Valentina ALMEIDA ${ }^{1}$

Acta Med Port 2018 May;31(5):254-264 - https://doi.org/10.20344/amp.10094

\section{RESUMO}

Introdução: O objectivo deste estudo é identificar o número de anestesiologistas e monitorizar a actividade da Anestesiologia em Portugal, comparando-a com idêntico Censos realizado em 2014.

Material e Métodos: Estudo observacional transversal. Foram contactados, em maio de 2017, os diretores dos serviços de Anestesiologia das instituições hospitalares com actividade cirúrgica que compõem o Serviço Nacional de Saúde, num total de 53 instituições hospitalares, correspondendo a 86 hospitais.

Resultados: O Censos registou um total de 615127 intervenções cirúrgicas (mais 3,4\% que em 2013), das quais $84,1 \%$ foram cirurgias programadas e destas 49,6\% em regime de ambulatório (mais 6,1\% que em 2013). Constatou-se ainda a realização de 89608 procedimentos com apoio de anestesia fora do bloco operatório (menos 19,0\% que em 2013), 282944 consultas de anestesia (mais 1,3\% que em 2013) e 112183 consultas de dor crónica (mais 13,1\% que em 2013). Identificaram-se 51380 analgesias de parto (mais $14,3 \%$ que em 2013), que corresponderam a 70,5\% dos partos ocorridos nos serviços de Obstetrícia dos hospitais do Serviço Nacional de Saúde (mais 5\% de partos que em 2013). Foram identificados 1280 Anestesiologistas (mais 2,1\% do que em maio de 2014), o que corresponde a um rácio de 12,4 por 100000 habitantes (este valor era de 12,0 em maio de 2014). Com os 262 Anestesiologistas a trabalhar exclusivamente no sector privado, obtém-se um total nacional de 1542 Anestesiologistas, ou seja, um rácio de 15,1 por 100 000 habitantes (este valor era 13,9 em maio de 2014).

Discussão: Prevê-se que o déficit de 541 Anestesiologistas identificados, possa ser reduzido em cerca de dois terços até 2023. A redução do déficit de Anestesiologistas permitirá aumentar a capacidade de resposta às crescentes solicitações dos serviços de anestesiologia Portugueses.

Conclusão: Apesar de um ligeiro aumento do rácio de anestesiologistas por habitante em 2017 comparativamente a 2014, Portugal continua com um déficit de Anestesiologistas.

Palavras-chave: Anestesiologia/recursos humanos; Anestesiologistas; Portugal

\section{ABSTRACT}

Introduction: The objective of this study is to identify the number of anaesthesiologists working in Portugal and to monitor the national activity of this medical specialty by comparing it with a similar Census performed in 2014.

Material and Methods: Observational cross-sectional study. Data related to the month of May 2017 was collected from Anaesthesiology departments of 53 Portuguese public institutions from a total of 86 hospitals.

Results: The Census registered 615127 surgical procedures (3.4\% more than in 2013$), 84.1 \%$ of which on a non-emergent basis, and $49.6 \%$ day case surgery (6.1\% more than in 2013). Moreover, 89608 procedures were performed outside the operating rooms (19.0\% less than in 2013), 282944 were anaesthetic clinics (1.3\% more than in 2013) and 112183 were chronic pain evaluations (13.1\% more than in 2013). In addition, 51380 labour analgesia were performed for delivery (14.3\% more than in 2013) corresponding to $70.5 \%$ of all deliveries occurring in the Obstetric department of Portuguese public hospitals in 2016 (5\% more than in 2013$)$. A total of 1280 Anaesthesiologists were identified (2.1\% more than in May 2014), corresponding to a ratio of 12.4 per 100000 inhabitants (it was 12.0 in May 2014). Together with the 262 anaesthesiologists that work exclusively in the private system, we found a total sum of 1542 anaesthesiologists indicating a ratio of 15.1 per 100000 inhabitants (it was 13.9 in 2014).

Discussion: We predict that the identified deficit of 541 anaesthesiologists in the Portuguese National Health Service should be reduced by two thirds until 2023. The reduction of the shortage of anaesthesiologists will allow an increase in human resource capacity in Anaesthesiology.

Conclusion: Even though there was a slight increase in the ratio of Anaesthesiologists per inhabitant in 2017 compared to 2014 , Portugal maintains a shortage of Anaesthesiologists.

Keywords: Anesthesiology/manpower; Anesthesiologists; Portugal

\section{INTRODUÇÃO}

A Anestesiologia é uma especialidade transversal à organização médica contemporânea, não só pela insubstituível intervenção na Medicina Hospitalar, como também pelo seu crescente apoio à Medicina Geral e Familiar, em áreas como a Medicina da Dor e a Medicina Paliativa.

Mas é na vertente hospitalar que mais se faz sentir a necessidade e premência da prestação de cuidados anes- tésicos:

a) Na Medicina Peri-Operatória, com a criação de condições indispensáveis à realização de procedimentos de elevada qualidade e segurança;

b) Na Medicina de Urgência e na Emergência Pré e Intra-Hospitalar, onde a integração e liderança da Anestesiologia em equipas multidisciplinares e

1. Direção do Colégio de Anestesiologia (2015 - 2017). Ordem dos Médicos. Lisboa. Portugal.

$\triangle$ Autor correspondente: Paulo Lemos. pauloferreiralemos@gmail.com

Recebido: 17 de dezembro de 2017 - Aceite: 23 de abril de 2018 | Copyright @ Ordem dos Médicos 2018 
multiprofissionais é objeto de reconhecimento;

c) Na Medicina Intensiva, no apoio ao doente crítico, nos cuidados diferenciados no pós-operatório de doentes submetidos a cirurgia altamente diferenciada ou ainda de doentes vítimas de grande trauma, onde diferentes saberes integradores e aquisição de múltiplas competências tornam a presença do Anestesiologista indispensável;

d) No apoio à realização de meios complementares de diagnóstico e terapêutica (MCDT), que apresentam complexidade crescente, exigindo a presença do Anestesiologista;

e) Na Medicina da Dor, quer em doentes agudos quer naqueles em que a dor se tornou um problema crónico, ou ainda na analgesia de parto ajudando as mulheres a experienciar sem dor um dos momentos mais gratificantes das suas vidas.

Todo este esforço de intervenção exige uma prestação de cuidados de crescente segurança e qualidade, permitindo uma melhoria dos resultados da recuperação funcional e da reintegração social, profissional e familiar dos doentes.

Esta evolução da especialidade carece de uma monitorização e avaliação periódica da organização dos serviços de Anestesiologia, assim como da necessidade de se conhecerem com rigor os recursos humanos existentes, de forma a conseguir-se um planeamento estratégico da especialidade e da necessária renovação ou acréscimo dos seus quadros médicos.

Esse trabalho iniciado em 2014, ${ }^{1}$ tem agora continuidade pela repetição do Censos Anestesiologia 2017, tendo como objetivo a atualização do conhecimento dos recursos humanos em Anestesiologia existentes no país, assim como da sua atividade, nas instituições hospitalares $(\mathrm{IH})$ do Serviço Nacional de Saúde (SNS).

\section{MATERIAL E MÉTODOS}

Os dados recolhidos resultaram dum inquérito que foi enviado por correio electrónico (Apêndice 01: https://www. actamedicaportuguesa.com/revista/index.php/amp/editor/ proofGalley/10094/5430) a todos os diretores dos serviços de Anestesiologia das IH com actividade cirúrgica que compõem o SNS (com modelos de gestão empresarial ou privada, no continente e regiões autónomas dos Açores e da Madeira, incluindo os três centros regionais do Instituto Português de Oncologia Dr. Francisco Gentil (IPOFG) e as duas unidades dos Hospitais das Forças Armadas (HFA) de Lisboa e Porto, num total de $53 \mathrm{IH}$, correspondendo a 86 Hospitais. Foi solicitado que os dados relativos à organização, recursos humanos e logística reportassem à semana entre 14 e 21 de maio de 2017, e os dados de produção ao ano de 2016.

Relativamente à caracterização da logística, foram solicitados dados sobre a tipologia hospitalar, especialidades cirúrgicas existentes, existência e caracterização do programa cirúrgico em regime de ambulatório, MCDT com intervenção da Anestesiologia e existência e caracterização do SU. Em relação aos recursos humanos em Anestesiolo- gia obtiveram-se dados demográficos sobre o número total de especialistas, a sua categoria profissional e o seu regime contratual, assim como dados relativos aos internos em formação. Foi ainda solicitado que os diretores de serviço identificassem os deficits existentes em recursos humanos, explicando a metodologia utilizada para a obtenção desses dados. Em relação à organização dos Serviços de Anestesiologia, solicitaram-se dados relativos à caracterização das tarefas diárias contabilizado o tempo despendido por períodos de seis horas. Finalmente, e em relação à produção dos serviços de Anestesiologia, foram solicitados dados anuais relativos aos atos anestésicos para cirurgia programada (tendo em conta o regime cirúrgico convencional versus ambulatório), para cirurgia de urgência, atos anestésicos praticados fora do bloco operatório (incluindo a analgesia de parto), e consultas de Anestesia e de Medicina da Dor.

Uma versão simplificada do inquérito (apenas com caracterização da logística existente no hospital e identificação nominal dos Anestesiologistas envolvidos) foi enviada por correio postal aos diretores clínicos dos hospitais privados com actividade cirúrgica, com a intenção de identificar todos os Anestesiologistas a trabalhar única e exclusivamente nesses hospitais. Finalmente, e para aumentar a fiabilidade do Censos, enviou-se uma mensagem por correio electrónico a todos os médicos inscritos no Colégio de Anestesiologia da Ordem dos Médicos (OM) solicitando àqueles que trabalhassem exclusivamente no sector privado à data de maio de 2017, que devolvessem uma resposta que incluísse o nome clínico, número de inscrição na OM e tempo de atividade profissional como Anestesiologista.

\section{RESULTADOS}

No presente projeto foram incluídas as $53 \mathrm{IH}$ com atividade cirúrgica, representando 47 no Continente, três na Região Autónoma dos Açores (Hospital do Divino Espírito Santo em Ponta Delgada, Hospital do Santo Espírito em Angra do Heroísmo e o Hospital da Horta), um na Região Autónoma da Madeira (Hospital Central Dr. Nélio Mendonça no Funchal), e dois das Forças Armadas (os Polos de Lisboa e Porto). Das $47 \mathrm{IH}$ do Continente, 20 são hospitais, 19 são centros hospitalares e oito integram unidades locais de saúde - ULS (Alto Minho, Matosinhos, Nordeste, Guarda, Castelo Branco, Norte Alentejano, Baixo Alentejo e Litoral Alentejano), sendo distribuídos geograficamente pela Administração Regional de Saúde (ARS) do Norte (15), Centro (11), Lisboa e Vale do Tejo (15), Alentejo (4) e Algarve (2).

As únicas diferenças relativamente ao Censos de 2014 residiram:

- Na separação do Serviço de Anestesiologia do Centro Hospitalar de Setúbal, que foi dividido em dois, ficando um serviço no Hospital de Setúbal e outro no Hospital de Outão;

- Na solicitação de dados mais pormenorizados na área de Obstetrícia. 


\section{Caracterização das instituições hospitalares do SNS}

As IH objeto desta análise apresentaram enormes diferenças na sua dimensão, especialidades cirúrgicas envolvidas e complexidade cirúrgica. A cirurgia eletiva é cada vez mais realizada em regime de ambulatório. Em 40 das $\mathrm{IH}$ analisadas $(75,5 \%)$ encontra-se organizado um modelo autónomo, isto é, com um circuito e instalações independentes do internamento (bloco operatório e área de recobro).

A envolvência da Anestesiologia em ambiente fora do bloco operatório é cada vez maior, sendo hoje frequente encontrar Anestesiologistas a dar apoio na área da Gastrenterologia $(90,6 \%$ das $\mathrm{IH})$, da Cardiologia de Intervenção e Pneumologia (75,5\%), Neurorradiologia (71,7\%), Radiologia $(54,7 \%)$ e ORL $(47,2 \%)$. De forma menos expressiva encontra-se o apoio à Psiquiatria (22,6\%) e à Medicina da Reprodução (18,9\%).

Apenas quatro IH não dispõem de SU aberto ao exterior (IPOFG Centro Regional de Coimbra, Hospital de Cantanhede, Hospital do Outão e Instituto de Oftalmologia Dr. Gama Pinto, em Lisboa). As 49 restantes apresentam complexidade diversa e uma distinta carteira de serviços, com vias verdes (AVC e Coronária) em 75,5\%, e valências especiais (Neurocirurgia, Vascular e Cardiotorácica) em 34,0\%. De salientar o elevado número de SU que mantêm atividade Obstétrica $(79,2 \%)$ e de Cirurgia Pediátrica (39,6\%).

$A$ atividade dos Anestesiologistas não se restringe ao bloco operatório e ao apoio à realização de MCDT.
Na verdade, e de acordo com as suas competências, no âmbito da Medicina Peri-Operatória, Medicina da Dor, Medicina Intensiva e de Emergência, o Anestesiologista é envolvido num conjunto de programas e tarefas exigentes que muito o absorvem. Realça-se a sua presença na totalidade das IH incluídas, no âmbito da Consulta de Anestesia, na Unidade de Cuidados Pós-Anestésicos e na Analgesia de Parto, quando existe serviço de Obstetrícia. Por outro lado, os Anestesiologistas têm actividade no âmbito da Dor Crónica em 84,9\% das IH, da Emergência Hospitalar em $73,6 \%$, em programas de Dor Aguda em $71,7 \%$, e na Medicina Intensiva em 58,1\%.

\section{Caracterização dos serviços de Anestesiologia e da sua atividade}

Trinta e sete serviços de Anestesiologia estão dependentes directamente da direcção clínica das $\mathrm{IH}$ analisadas $(69,8 \%)$, estando seis integradas em departamentos com a Medicina Intensiva $(11,3 \%)$ e oito em departamentos de Cirurgia (15,1\%). Numa $\mathrm{IH}$, a Anestesiologia passou a ser uma unidade funcional, e noutra dado a sua inexistência, estes serviços médicos estão a cargo de uma empresa externa de prestação de serviços de Anestesiologia.

As múltiplas funções dos Anestesiologistas e a sua resposta às diferentes solicitações em ambiente hospitalar, foram obtidas considerando períodos de tempo semanais com a duração de seis horas. Dos resultados apurados,

Tabela 1 - Produção anual (2016 e 2013) nas instituições hospitalares públicas analisadas, com relação à atividade anestésica

\begin{tabular}{|c|c|c|c|c|}
\hline \multirow{2}{*}{ Produção anual } & \multicolumn{2}{|c|}{$2016(n=53)$} & \multicolumn{2}{|c|}{$2013(n=52)$} \\
\hline & $n^{\circ}(\%)$ & Média \pm DP & $n^{\circ}(\%)$ & Média \pm DP \\
\hline $\begin{array}{l}\text { Total de cirurgia } \\
\text { programada }\end{array}$ & $\begin{array}{c}517058 \\
(84,06 \%)\end{array}$ & $9755,8 \pm 8717,3$ & $\begin{array}{c}492784 \\
(82,80 \%)\end{array}$ & $9476,6 \pm 7968,6$ \\
\hline $\begin{array}{l}\text { Total de cirurgia em } \\
\text { regime de internamento }\end{array}$ & $\begin{array}{c}260693 \\
(50,42 \%)\end{array}$ & $4918,7 \pm 4824,2$ & $\begin{array}{c}278358 \\
(56,49 \%)\end{array}$ & $5353,0 \pm 4776,9$ \\
\hline $\begin{array}{l}\text { Total de cirurgia em } \\
\text { regime de ambulatório }\end{array}$ & $\begin{array}{c}256365 \\
(49,58 \%)\end{array}$ & $4837,1 \pm 4455,1$ & $\begin{array}{c}214426 \\
(43,51 \%)\end{array}$ & $4123,6 \pm 3772,1$ \\
\hline Total de cirurgia urgente & $\begin{array}{c}98069 \\
(15,94 \%)\end{array}$ & $1850,4 \pm 1550,2$ & $\begin{array}{c}102401 \\
(17,20 \%)\end{array}$ & $2007,9 \pm 1765,5$ \\
\hline $\begin{array}{l}\text { Total de intervenções } \\
\text { efectuadas }\end{array}$ & 615127 & $11606,2 \pm 10013,0$ & 595185 & $11445,9 \pm 9509,7$ \\
\hline $\begin{array}{l}\text { Fora do bloco operatório } \\
\text { (locais remotos) }\end{array}$ & 89608 & $1792,2 \pm 4565,3$ & 110668 & $2258,5 \pm 6259,6$ \\
\hline Total de partos & 72902 & $1375,5 \pm 1115,0$ & & \\
\hline Taxa de cesariana (\%) & $27,73 \%$ & & & \\
\hline $\begin{array}{l}\text { Hospitais com taxa de } \\
\text { cesariana inferior a } 27 \%\end{array}$ & 14 & & & \\
\hline $\begin{array}{l}\text { Total de analgesias de } \\
\text { parto }\end{array}$ & $\begin{array}{c}51380 \\
(70,48 \%)\end{array}$ & $969,4 \pm 856,6$ & 44956 & $881,5 \pm 789,0$ \\
\hline $\begin{array}{l}\text { Total de consultas de } \\
\text { anestesia* }\end{array}$ & $\begin{array}{c}282944 \\
(54,36 \%)\end{array}$ & $5338,6 \pm 4620,6$ & $\begin{array}{c}279205 \\
(56,66 \%)\end{array}$ & $5474,6 \pm 4835,2$ \\
\hline $\begin{array}{l}\text { Total de consultas de dor } \\
\text { crónica }\end{array}$ & 112183 & $2116,7 \pm 2056,7$ & 99153 & $1983,1 \pm 1841,1$ \\
\hline $\begin{array}{l}\text { Total de doentes } \\
\text { observados em dor aguda }\end{array}$ & 65562 & $1260,8 \pm 1771,8$ & 68858 & $1350,2 \pm 1924,7$ \\
\hline
\end{tabular}

* o valor percentual representa o $\mathrm{n}^{\circ}$ de consultas em relação à cirurgia programada efectuada

DP: desvio padrão 
salienta-se que a média nacional de atividade cirúrgica programada é de 7,8 salas operatórias por dia útil e por $\mathrm{IH}$, sendo 3/4 ocupadas durante a manhã e 1/4 à tarde.

A atividade no SU representa cerca de $32 \%$ de toda a atividade anestésica, enquanto a que é dedicada fora do bloco operatório (consultas, apoio à realização de MCDT, Dor, Cuidados Intensivos, Emergência, Ensino, etc.) se encontra em crescimento, representando mais de $35 \%$ do total.

No que respeita à produção hospitalar relativa ao ano de 2016, descriminam-se alguns dados na Tabela 1. O Censos registou um total de 615127 intervenções cirúrgicas realizadas nas $53 \mathrm{IH}$ analisadas, das quais $84,1 \%$ foram cirurgias programadas e destas $49,6 \%$ em regime de ambulatório. Salienta-se uma produção cirúrgica global de $3,4 \%$ superior do que a registada em 2013 , com uma redução da cirurgia urgente que é agora de 15,9\%. Houve também um aumento da cirurgia em regime de ambulatório que se situa agora perto dos $50 \%$. Dos 86254 partos registados em Portugal em 2016, 72902 (84,5\%) ocorreram nos serviços de Obstetrícia dos hospitais do SNS, 70,5\% dos quais sob analgesia. Contudo, como dos partos ocorridos nas IH do SNS cerca de 15\% são cesarianas programadas, poder-se-á especular que mais de $85 \%$ das grávidas que iniciaram trabalho de parto em IH do SNS beneficiaram de uma analgesia de parto durante o ano de 2016 (recorda-se que o valor calculado para 2013 foi de $80 \%$ ). Ainda na área obstétrica de assinalar a taxa de cesariana nacional nas $\mathrm{IH}$ do SNS de $27,73 \%$, e de registar que apenas um terço destes conseguiu obter uma taxa de cesariana inferior a $27 \%$. Em relação às médias, estas acabam por ter um interesse relativo dado a grande dispersão apresentada pelos resultados dos diferentes hospitais.

\section{Caracterização dos Recursos Humanos em Anestesio- logia}

A) Pertencentes aos quadros dos serviços de Anestesiologia das IH do SNS, independentemente do modelo de gestão

Das $53 \mathrm{IH}$ que fazem parte do SNS foram identificados como pertencentes aos quadros médicos dos serviços de Anestesiologia, na semana de 14 a 21 de maio de 2017, 1158 Anestesiologistas (mais 3,3\% que em 2014), dos quais 847 pertencem ao sexo feminino (73,1\%). Curiosamente em 2014 tal percentagem representava 73,8\%, denotando uma pequena inversão na evolução dos últimos anos.

Na Fig. 1 encontram-se estratificados os 1158 Anestesiologistas por data de nascimento. De notar que a média de idades obtida foi de 48,6 anos, demonstrando um ligeiro envelhecimento da população em estudo (havia sido de 47,9 anos em 2014).

B) Anestesiologistas das IH do SNS não pertencentes aos quadros dos serviços de Anestesiologia, independentemente do modelo de gestão

Para além dos 1158 Anestesiologistas, anteriormente referidos, e pertencentes aos quadros médicos dos serviços de Anestesiologia das $\mathrm{IH}$ analisadas, existem nestas, 76 Anestesiologistas autónomos dos serviços de Anestesiologia, e que pertencem sobretudo a unidades de cuidados intensivos ou a unidades de Dor Crónica. Estes Anestesiologistas são maioritariamente do sexo feminino (69,7\%).

C) Outros Anestesiologistas das IH do SNS, sem vínculo contratual

Para além dos 1234 Anestesiologistas anteriormente referidos, existiam ainda à data da realização deste Censos, 46 Anestesiologistas eventuais, que aguardavam colocação após a obtenção do grau de Assistente, dos quais $84,8 \%$ eram do sexo feminino.

\section{D) Internos de Formação Específica em Anestesio-} logia

Com a entrada em vigor da Portaria n 49/2011 de 26 de janeiro, que reviu a estrutura e organização do Internato de Anestesiologia, este passou a ter a duração de cinco anos. Por outro lado, a revisão do Regulamento do referido Internato publicada através da Portaria n 92-A/2016 de 15 de Abril, permitiu o alargamento da capacidade formativa nacional anual para valores que rondam os 80 internos de formação específica (IFE) em Anestesiologia. Assim, o Censos permitiu identificar 363 IFE em Anestesiologia, com uma predominância feminina $(67,2 \%)$. Acrescenta-se o facto da idoneidade formativa em Anestesiologia ter sido alargada nos últimos três anos, a mais três hospitais públicos (Hospital Faro (HF), Hospital Vila Franca de Xira (HVFX) e Hospital Beatriz Ângelo (HBA)), e a um privado (Hospital da Luz, em Lisboa).

E) Anestesiologistas a trabalhar exclusivamente em hospitais privados

Desde aposentados até jovens recém-especialistas existe um variado conjunto de Anestesiologistas que exerce atividade exclusiva em hospitais privados, num número que se encontra avaliado em 262 profissionais.

F) Rácio de Anestesiologistas por habitante e região

Dos resultados apresentados verifica-se a existência de 1280 Anestesiologistas com vínculo a IH do SNS (Tabela 2), com um rácio de 12,4 por 100000 habitantes que aumenta para 15,1 se incluirmos os 262 Anestesiologistas que trabalham exclusivamente no sector privado. Regista-se assim uma evolução positiva em quase todas as regiões de saúde. Não esquecer, contudo, que a diminuição da população residente em Portugal registada nos últimos dois anos ajudou também a melhorar este indicador.

Quando solicitados a informarem quais os déficits que os diferentes diretores dos serviços de Anestesiologia das IH do SNS identificam na gestão dos seus recursos humanos, e que procuram colmatar da mais diversa maneira, através de horas extraordinárias, contratação de Anestesiologistas tarefeiros, ou mesmo encerrando postos de 


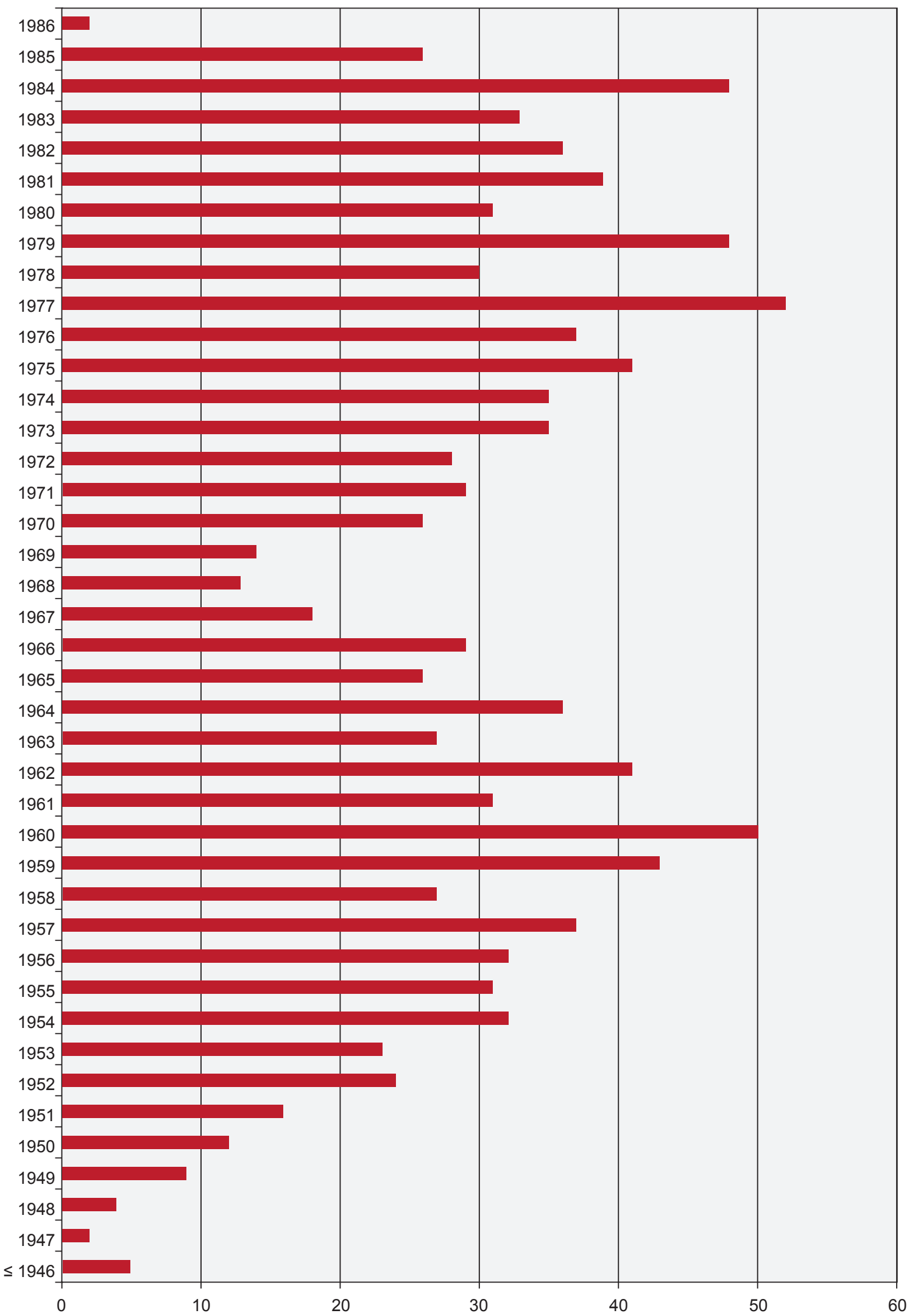

Figura 1 - Anestesiologistas pertencentes aos quadros médicos dos serviços de Anestesiologia das instituições hospitalares públicas analisadas $(n=53)$, por ano de nascimento 
rabalho, verificamos que o número relativamente aos Anestesiologistas do quadro, se agravou, passando de $41,7 \%$ em 2014 para 46,7\% em 2017 (Tabela 3).

Haverá muitas razões para este agravamento, mas seguramente as mais importantes serão:

- a saída não compensada de vários Anestesiologistas para hospitais privados e para o estrangeiro, pela maior competitividade destes;

- a aplicação aos médicos do SNS de legislação laboral existente em Lei há muitos anos, com descansos compensatórios com prejuízo de horário para aqueles que trabaIham em horário noturno ou em dias de descanso semanal;

- a contratação de novos Anestesiologistas com horários incluindo 18 horas de serviço de urgência, ao contrário dos anteriores horários que incluíam 12 horas de serviço de urgência (e que representam em 2017, 21,2\% dos Anestesiologistas dos quadros médicos, em comparação com os 7,9\% em 2014), levou a uma redução efetiva da carga horária disponível para trabalho programado em cerca de $30 \%$;

traduzindo-se todas estas alterações em significativas reduções da disponibilidade de recursos humanos médicos para dar resposta às diferentes solicitações do dia a dia.

Esclarece-se que estes valores são da única e exclusiva responsabilidade dos respectivos diretores de serviços de Anestesiologia, cuja larga maioria não se mostrou disponível para explicar como os obteve.

\section{Indicadores relacionados com a atividade da Aneste- siologia}

Neste estudo optamos por utilizar o agrupamento definido pela Administração Central do Sistema de Saúde (ACSS) no modelo proposto de monitorização do SNS, através da inclusão de diferentes dimensões de benchmarking, como sejam indicadores económico-financeiros, de acesso, de produção ou qualidade. ${ }^{3}$

Incluíram-se nesta análise apenas as IH dos Grupos C, D e E, em virtude dos restantes, quer seja por especificidades próprias ou essencialmente pelo baixo número de recursos humanos em anestesiologia sentido em muitos dos hospitais de tipo A e B, facilmente poderem enviesar os resultados obtidos.

Dos indicadores analisados e apresentados na Tabela
4, um deles procurou refletir a gestão de recursos humanos em Anestesiologia através do rácio de número de horas resultantes da carga horária semanal (procurando incluir períodos em horas extraordinárias ou períodos de trabalho de tarefeiros) dos diferentes profissionais sobre o número de horas previstas para o cumprimento das diversas tarefas solicitadas aos serviços de Anestesiologia (incluindo SU, mas excluindo períodos de trabalho de cirurgia adicional). Estas foram avaliadas numa perspectiva de período de seis horas, para facilitar a contabilização das horas atribuídas em geral à actividade em bloco operatório. Um valor igual a 1 representa o match perfeito entre a carga horária disponível e as diferentes solicitações a que os serviços devem corresponder. Se o valor for superior a 1 , significa que existe algum desperdício na gestão de recursos humanos, e se inferior, significará que para conseguir dar resposta, o serviço estará a ser muito eficiente ou se esqueceu de incluir horas contratualizadas para que possam corresponder efetivamente às solicitações que são colocadas aos serviços de Anestesiologia (coluna 'rácio horas $\mathrm{RH}$ / períodos'). $\mathrm{Na}$ Tabela 4 encontram-se ainda identificados vários indicadores da actividade em que os anestesiologistas estão envolvidos, como por exemplo, a percentagem de consultas de Anestesia realizadas nos doentes propostos para cirurgia programada das diferentes $\mathrm{IH}$, a percentagem de cirurgia em regime de ambulatório no total de cirurgia programada realizada, o número de consultas de Anestesia por período de seis horas, o número de consultas de Dor Crónica por período de seis horas, o número de cirurgias por tempo operatório eletivo, o número de intervenções sob anestesia fora do bloco operatório por período de seis horas, e o número de anestesias por sala operatória dedicada ao SU. Existem alguns dados que se destacam pela sua improbabilidade, como por exemplo 35,2 consultas por período de seis horas, ou 22,9 procedimentos anestésicos fora do bloco operatório por período de seis horas, o que daria uma consulta ou um procedimento anestésico por cada 10 e 15 min, respectivamente! Estes fatos podem também ser devidos a falhas de registo.

É de salientar a tendência decrescente do número de consultas por cirurgia programada (de $57,1 \%$ para $39,1 \%$ ), da percentagem de cirurgia ambulatória (de 53,5\% para $42,7 \%$ ), do número de consultas por período de seis horas

Tabela 2 - Rácio Anestesiologista por 100000 habitantes, por Administração Regional de Saúde em 2017 e 2014

\begin{tabular}{|c|c|c|c|c|}
\hline \multirow[b]{2}{*}{ Administrações Regionais de Saúde } & \multirow[b]{2}{*}{ Anestesiologistas } & \multirow[b]{2}{*}{ População* } & \multicolumn{2}{|c|}{ or 100000 hab } \\
\hline & & & 2017 & 2014 \\
\hline Norte & 525 & 3603778 & 14,6 & 13,5 \\
\hline Centro & 225 & 2256364 & 10,0 & 9,7 \\
\hline Lisboa e Vale do Tejo & 429 & 2812678 & 15,3 & 15,7 \\
\hline Alentejo & 28 & 724391 & 3,9 & 3,8 \\
\hline Algarve & 20 & 441929 & 4,5 & 5,7 \\
\hline Região Autónoma dos Açores & 30 & 245766 & 12,2 & 9,7 \\
\hline Região Autónoma da Madeira & 23 & 256424 & 9,0 & 8,0 \\
\hline Total & 1280 & 10341330 & 12,4 & 12,0 \\
\hline
\end{tabular}

${ }^{*}$ residente em Portugal a 31 de dezembro de 2015, segundo o Instituto Nacional de Estatística (INE) 
Tabela 3 - Déficits de Anestesiologistas em 2017 por hospital, da responsabilidade do diretor de serviço de Anestesiologia

\begin{tabular}{|c|c|c|c|c|}
\hline Região & Hospitais & $\begin{array}{c}\text { Anestesiologistas } \\
\text { Serviço }\end{array}$ & Deficit & $\%$ \\
\hline \multirow{15}{*}{ ARS Norte } & $\mathrm{CH}$ Alto Minho & 16 & 6 & $37,5 \%$ \\
\hline & H Braga & 43 & 8 & $18,6 \%$ \\
\hline & H Barcelos & 5 & 1 & $20,0 \%$ \\
\hline & $\mathrm{CH}$ Alto Ave & 18 & 5 & $27,8 \%$ \\
\hline & CH Médio Ave & 14 & 6 & $42,9 \%$ \\
\hline & CH Póvoa - Vila do Conde & 11 & 5 & $45,5 \%$ \\
\hline & CH Tâmega - Sousa & 26 & 9 & $34,6 \%$ \\
\hline & CHTMAD & 15 & 10 & $66,7 \%$ \\
\hline & CH Nordeste & 10 & 9 & $90,0 \%$ \\
\hline & ULS Matosinhos & 36 & 8 & $22,2 \%$ \\
\hline & CH S João & 71 & 15 & $21,1 \%$ \\
\hline & IPO Porto & 24 & 6 & $25,0 \%$ \\
\hline & CH Porto & 77 & 15 & $19,5 \%$ \\
\hline & CH Gaia - Espinho & 57 & 10 & $17,5 \%$ \\
\hline & $\mathrm{CH}$ Entre Douro e Vouga & 24 & 8 & $33,3 \%$ \\
\hline \multirow{10}{*}{ ARS Centro } & CH Baixo Vouga & 19 & 12 & $63,2 \%$ \\
\hline & $\mathrm{CH}$ Tondela - Viseu & 33 & 16 & $48,5 \%$ \\
\hline & H Cantanhede & 0 & 2 & \\
\hline & H Figueira da Foz & 8 & 12 & $150,0 \%$ \\
\hline & CH Universitário Coimbra & 92 & 38 & $41,3 \%$ \\
\hline & IPO Coimbra & 11 & 2 & $18,2 \%$ \\
\hline & ULS Guarda & 6 & 3 & $50,0 \%$ \\
\hline & $\mathrm{CH}$ Cova da Beira & 8 & 8 & $100,0 \%$ \\
\hline & ULS Castelo Branco & 9 & 3 & $33,3 \%$ \\
\hline & CH Leiria - Pombal & 18 & 6 & $33,3 \%$ \\
\hline \multirow{7}{*}{ ARS Lisboa } & $\mathrm{CH}$ Oeste & 15 & 6 & $40,0 \%$ \\
\hline & H Santarém & 9 & 11 & $122,2 \%$ \\
\hline & H V F Xira & 19 & 3 & $15,8 \%$ \\
\hline & H Beatriz Ângelo & 29 & 3 & $10,3 \%$ \\
\hline & H Fernando Fonseca & 26 & 8 & $30,8 \%$ \\
\hline & $\mathrm{CH}$ Cascais & 19 & 3 & $15,8 \%$ \\
\hline & CH Lisboa Ocidental & 57 & 16 & $28,1 \%$ \\
\hline e & CH Lisboa Central & 94 & 44 & $46,8 \%$ \\
\hline \multirow{7}{*}{ Vale do Tejo } & CH Lisboa Norte & 49 & 49 & $100,0 \%$ \\
\hline & Instituto Oftalmologia Gama Pinto & 4 & 2 & $50,0 \%$ \\
\hline & IPO Lisboa & 20 & 4 & $20,0 \%$ \\
\hline & CH Barreiro - Montijo & 10 & 7 & $70,0 \%$ \\
\hline & H Garcia de Orta & 20 & 21 & $105,0 \%$ \\
\hline & CH Setúbal & 13 & 8 & $61,5 \%$ \\
\hline & H Outão & 3 & 2 & $66,7 \%$ \\
\hline \multirow{4}{*}{ ARS Alentejo } & H Évora & 13 & 7 & $53,8 \%$ \\
\hline & ULS Norte Alentejano & 5 & 11 & $220,0 \%$ \\
\hline & ULS Baixo Alentejo & 4 & 8 & $200,0 \%$ \\
\hline & H Litoral Alentejano & 5 & 14 & $280,0 \%$ \\
\hline \multirow{2}{*}{ ARS Algarve } & H Faro & 12 & 18 & $150,0 \%$ \\
\hline & H Barlavento Algarvio & 6 & 8 & $133,3 \%$ \\
\hline RA Madeira & HC Funchal & 21 & 28 & $133,3 \%$ \\
\hline \multirow{3}{*}{ RA Açores } & H Ponta Delgada & 16 & 8 & $50,0 \%$ \\
\hline & H Terceira & 8 & 4 & $50,0 \%$ \\
\hline & H Horta & 4 & 1 & $25,0 \%$ \\
\hline Hospitais Militares & Polo Lisboa & 10 & 4 & $40,0 \%$ \\
\hline
\end{tabular}


Tabela 4 - Indicadores de gestão de recursos humanos e de produção da atividade anestesiológica nos hospitais do Grupo C, D e E, em 2016

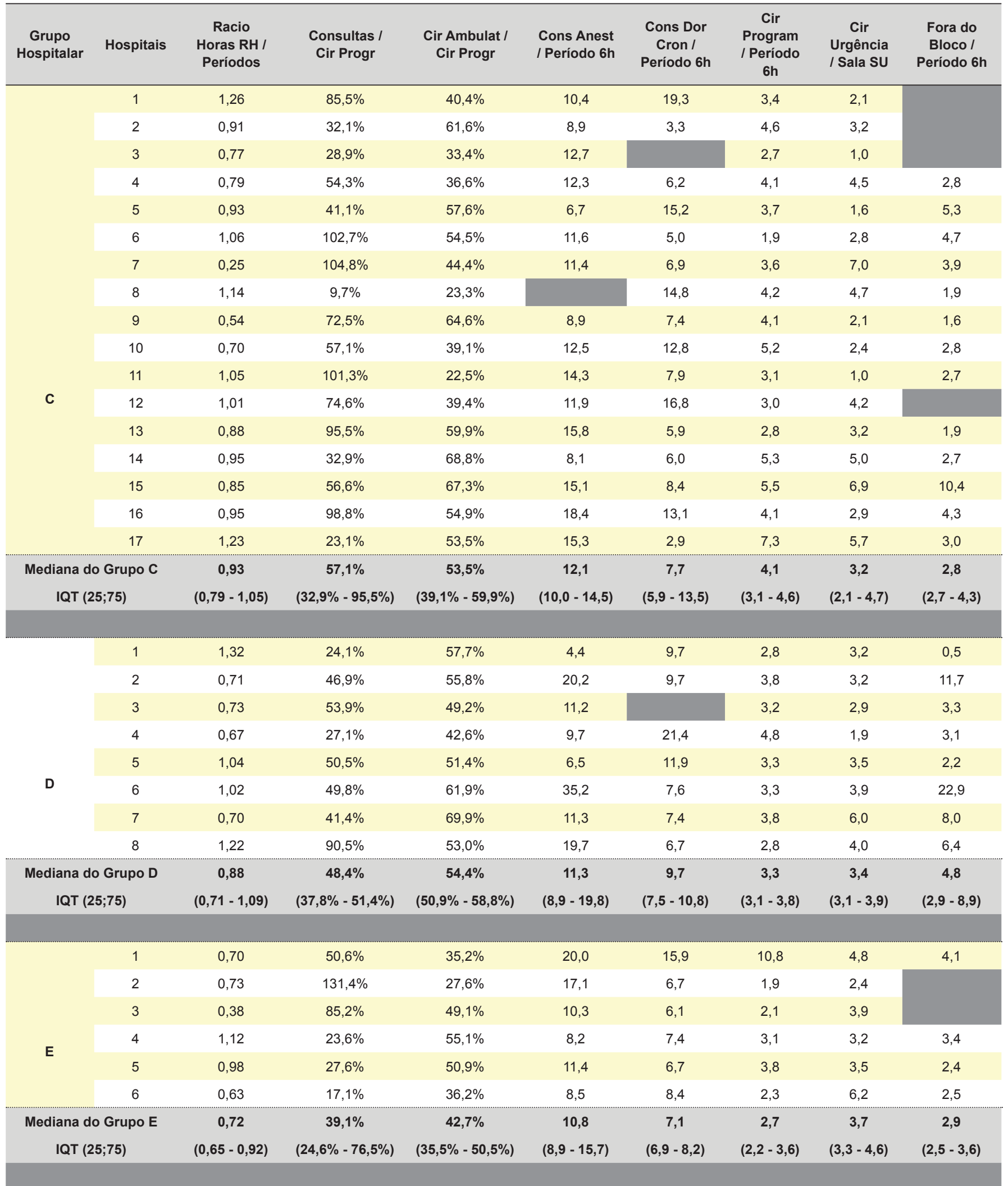

Grupo C: CH Alto Minho, CH Alto Ave, CH Tâmega-Sousa, ULS Matosinhos, CH Entre Douro e Vouga, CH Cova da Beira, CH Leiria-Pombal, CH Médio Tejo, H Santarém, CH Cascais PPP, H Beatriz Ângelo, CH Barreiro-Montijo, CH Setúbal, ULS Norte Alentejano, ULS Baixo Alentejo e H Barlavento Algarvio

Grupo D: H Braga, CH Trás-os-Montes e Alto Douro, CH Gaia-Espinho, CH Tondela-Viseu, H Fernando da Fonseca, H Garcia de Orta, H Évora e H Faro

Grupo E: CH S João, CH Porto, CH Universitário de Coimbra, CH Lisboa Ocidental, CH Lisboa Central e CH Lisboa Norte

IQT: intervalo interquartil 
(de 12,1 para 10,8), do número de cirurgias programadas por período de seis horas (de 4,1 para 2,7), nos diferentes grupos de hospitais à medida que aumenta a complexidade e diversidade de serviços prestados pelo hospital (de C para E). Por outro lado, salientar a tendência inversa do número de cirurgias urgentes por sala operatória adstrita ao serviço de urgência (de 3,2 para 3,7).

As diferentes $\mathrm{IH}$ encontram-se codificadas, sendo cada código do conhecimento exclusivo do respetivo diretor de serviço de Anestesiologia, que pode assim comparar os seus indicadores em relação à média do grupo em que a sua $\mathrm{IH}$ se encontra inserido.

\section{DISCUSSÃO}

A existência de serviços hospitalares autónomos constitui um elemento essencial para a manutenção de elevados padrões de qualidade no exercício clínico, não só por permitirem a aprendizagem recíproca e a formação contínua ao longo da vida profissional, como também por serem a forma privilegiada de se estabelecerem programas de avaliação e melhoria clínica, baseados numa colaboração e confiança interpares.

Assim, e pela transversalidade da intervenção da Anestesiologia na organização hospitalar, é recomendado que os serviços de Anestesiologia sejam autónomos, independentes e únicos por $\mathrm{IH}$, como aliás acontece. Se tal não for a opção do conselho de administração, então, mantendo a sua independência orgânica, é desejável que o serviço seja inserido em estruturas de gestão intermédia, com serviços cuja produtividade não esteja diretamente dependente da disponibilidade dos Anestesiologistas (como é exemplo a Medicina Intensiva).

Dos resultados obtidos é possível inferir-se que:

a) Se registou uma maior atividade cirúrgica programada, em especial em regime de ambulatório;

b) Houve menos procedimentos com apoio de Anestesia fora do bloco operatório possivelmente devido aos déficits de especialistas em Anestesiologia e à estratégia seguida pelos hospitais de privilegiar a actividade cirúrgica, economicamente mais rentável e com maior impacto sobre as expectativas e pressão da Sociedade;

c) Se conseguiu tratar um maior número de grávidas com o objetivo de aumentar a segurança, qualidade e o conforto das futuras mães durante o trabalho de parto, sendo hoje Portugal um país no contexto europeu com valores de analgesia de parto muito acima da média europeia ${ }^{4}$;

d) Se reduziu a percentagem de doentes com consulta de Anestesia, facto bem negativo se tivermos em linha de conta que se perde a oportunidade para optimização clínica (quando tal é necessário), a avaliação do risco peri-operatório, o esclarecimento e informação dos doentes, o planeamento antecipado da estratégia peri-operatória com vista a melhores resultados no pós-operatório.

e) Se agravaram os deficits de Anestesiologia identifi- cados pelos diretores de serviço de Anestesiologia das instituições hospitalares do SNS, muito também em virtude da implementação de regras laborais que visam a promoção de segurança no trabalho mas que retiraram tempo de trabalho efetivo, como seja a aplicação legal dos descansos compensatórios com prejuízo de horário.

A Anestesiologia tem sido uma especialidade em ampla expansão nas últimas duas décadas, adquirindo novas competências e assumindo novos desafios, acompanhando a evolução da ciência médica em geral. Assim, parece natural as necessidades crescentes destes especialistas, em especial nas IH do SNS.

No Censos de 2014, identificaram-se 1192 Anestesiologistas nos quadros médicos das IH do SNS. Entretanto nos últimos três anos, formaram-se mais 145 especialistas dos quais $99(68,3 \%)$ terão ingressado nos quadros médicos das IH do SNS. Ou seja, um terço dos jovens especialistas em Anestesiologia opta por não celebrar contrato com as IH do SNS. No Censos de 2014 já chamávamos a atenção para a importância de se aumentar a capacidade formativa em Anestesiologia. A reestruturação do Internato de Anestesiologia, registada em 2016 através da Portaria $n^{\circ}$ 92-A/2016 de 15 de abril, veio permitir essa transformação, passando a capacidade formativa máxima nacional de Anestesiologistas a ser de 80 médicos em vez dos 64 possíveis pela legislação anterior. Contudo, se deste esforço apenas dois terços dos novos especialistas efetivamente celebram contrato com as IH do SNS dificilmente conseguiremos no tempo estimado de três anos aumentar em 300 Anestesiologistas como havia sido previsto no Censos de 2014. Aliás a projeção dada pela Fig. 2, que prevê um cenário de saídas dos actuais Anestesiologistas aos 66 anos por aposentação, com as entradas já previstas de IFE, diz-nos que só poderemos obter mais 200 Anestesiologistas aos actuais 1234 até 2020, bem aquém dos 1554 expectáveis no Censos 2014 para esse ano.

Contudo, esse cenário poderá ser bem diferente a partir dessa data, já que a curva prevista aproximar-se-á mais do cenário identificado como Balanço II na Fig.2, permitindo reduzir em cerca de dois terços até 2023 (à custa dos actuais 363 IFE em Anestesiologia, em formação) as necessidades apontadas pelos diretores de serviço de Anestesiologia das IH do SNS.

É curioso que o déficit de 541 Anestesiologistas nas IH do SNS, apontado pelos diferentes diretores dos serviços de Anestesiologia, esteja em linha com a previsão das necessidades calculadas segundo a metodologia utilizada na Rede Nacional de Especialidade Hospitalar e de Referenciação da Anestesiologia (documento aprovado pela tutela em Junho de 2017), cujo cálculo atribui aos hospitais, segundo a sua tipologia (I, II e III) o número de 20, 48 e 99 Anestesiologistas, respetivamente. ${ }^{5}$

O presente estudo permite concluir uma melhoria dos rácios de Anestesiologistas por 100000 habitantes, de 13,9 em maio de 2014 para 15,1 em 2017, mas muito longe dos 17,9 avançados pela World Federation of Societies of 


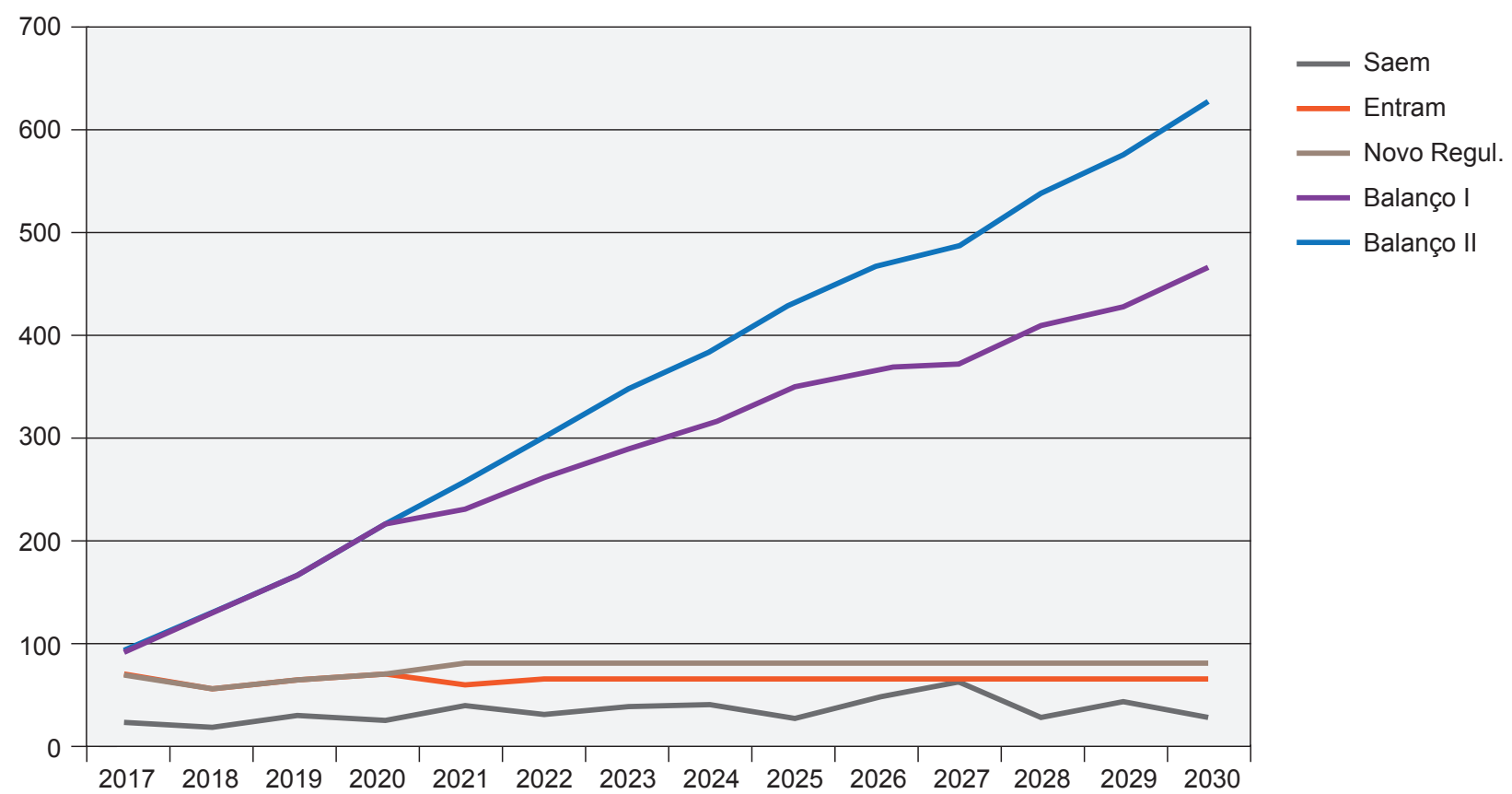

Figura 2 - Evolução dos Recursos Humanos em Anestesiologia até 2030 nas instituições hospitalares públicas analisadas, tendo em conta saídas aos 66 anos por aposentação e entradas fixas anuais de 64 e 80 internos, Balanço I e Balanço II, respetivamente

Anaesthesiologists (WFSA) para Portugal. (Fig. 3). ${ }^{6}$

Não existe um valor de referência internacional nesta matéria, mas a verdade é que a maioria dos países desenvolvidos (América do Norte, Austrália, Europa) apresentam valores em torno dos 20 Anestesiologistas por 100000 habitantes, sendo a Áustria e a Alemanha com 39,3 e 31,0, respectivamente, os países europeus com o mais elevado rácio de Anestesiologistas por habitante. ${ }^{6}$

Não temos, no entanto dificuldade, em perceber que existem deficits importantes de Anestesiologistas emPortugal, que importa corrigir nos próximos anos, de forma a dotar o nosso país dos Anestesiologistas necessários à prestação atempada e universal de cuidados anestésicos de elevada qualidade. Parece claro que, com a redução da mortalidade directamente relacionada com a Anestesiologia nas últimas décadas (1:200 000 anestesias) ${ }^{7}$ e com o aumento da segurança e qualidade da prestação de cuidados anestésicos, torna-se crucial avaliar a importância do alargamento da área de intervenção da nossa especialidade para áreas fora do bloco operatório, em especial no que toca à realização de MCDT com apoio da Anestesia, à Medicina da Dor, à Medicina de Emergência e Intensiva e,

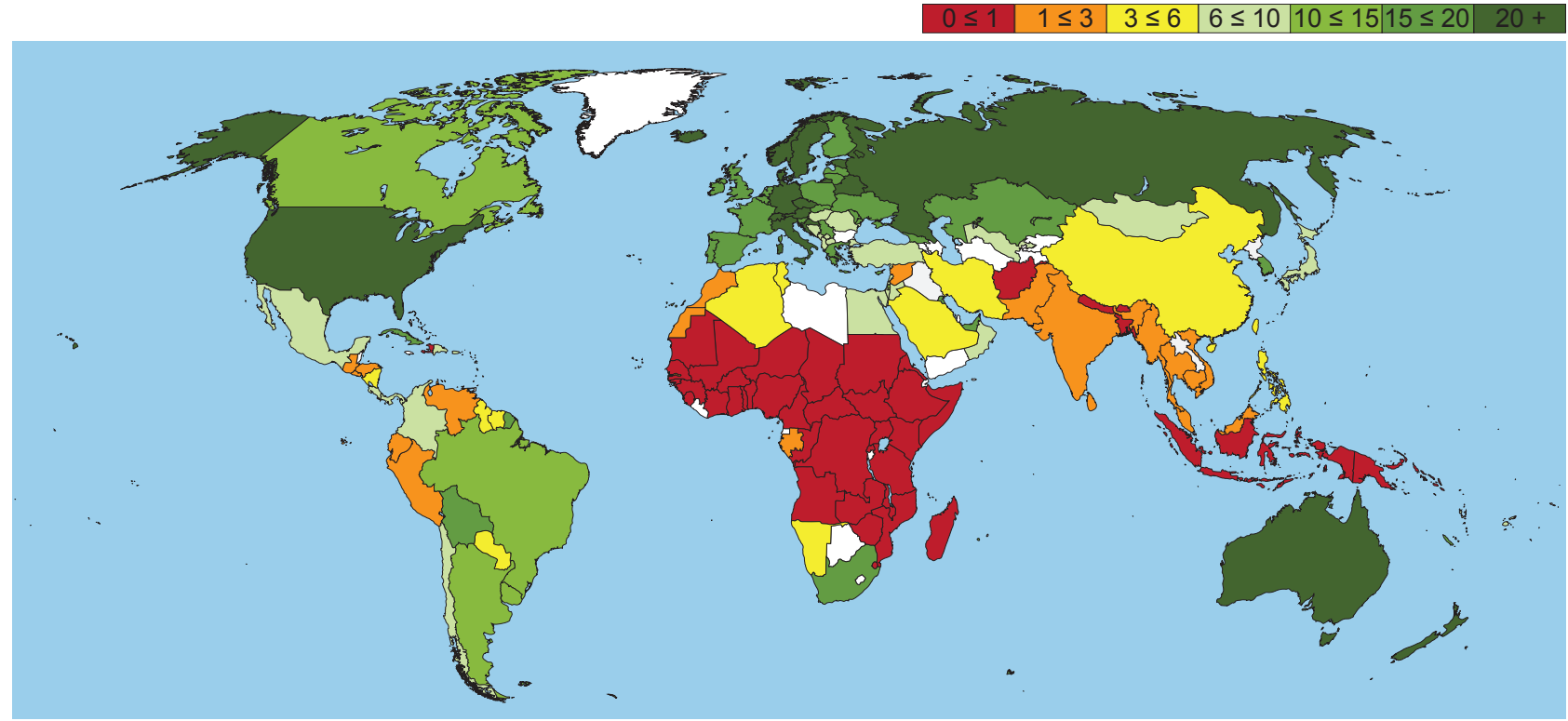

Figura 3 - Distribuição mundial de Anestesiologistas por 100.000 habitantes (países a branco sem informação disponível) (N.A.: Reprodução autorizada por Anesthesia \& Analgesia, publicada $\mathrm{em}^{6}$ e refeita em formato vectorial pela AMP) 
ainda, de forma muito particular nos cuidados pós-operatórios no âmbito da Medicina Peri-Operatória. Aliás, um dos enfoques actuais situa-se na mortalidade pós-operatória que apesar de toda a evolução técnica sentida na Medicina Moderna continua a ser uma importante preocupação de Saúde Pública, sobretudo quando percebemos fruto da análise de vários estudos que 30 a $50 \%$ dessas mortes serão evitáveis. ${ }^{8}$ Desta forma, permitiríamos não só uma previsível redução da morbi-mortalidade pós-cirúrgica, como uma recuperação funcional bem mais rápida, com níveis superiores de conforto e satisfação por parte dos nossos doentes.

Percebe-se também a importância crítica que pode ter a implementação imediata de medidas alternativas para minorar a curto prazo o déficit de Anestesiologistas, como sejam:

a) novos Contratos Individuais de Trabalho (CIT) para Anestesiologistas que não sejam abrangidos pelo atual enquadramento legislativo que impõe a atribuição de 18 horas da carga horária semanal para o SU, mas sim que permitam se necessário a atribuição de 12 ou menos horas da carga horária semanal para o SU. Salienta-se que os actuais CIT representam em comparação com os antigos uma redução de carga horária estimada em $30 \%$;

b) criar condições competitivas para os Anestesiologistas ficarem nos hospitais do SNS, em comparação com os hospitais privados ou aqueles que sendo públicos têm modelos de gestão privatizada.

Acreditamos assim que, com as múltiplas iniciativas sugeridas, se possa colmatar os déficits encontrados tão rápido quanto possível e, sobretudo, conseguir ter uma inversão da situação atual dos recursos humanos em Anestesiologia existentes no país, já em 2020. Deve referir-se a possibilidade de existência de viés no cálculo do rácio obtido, se os assistentes eventuais não forem contratados para os serviços de Anestesiologia das IH do SNS. Outra limita- ção do estudo foi a incapacidade dos autores conseguirem identificar a totalidade dos anestesiologistas que trabalham exclusivamente no sector privado.

\section{CONCLUSÃO}

O Censos registou um total de 615127 intervenções cirúrgicas realizadas nas $53 \mathrm{IH}$ analisadas, das quais $84,1 \%$ foram cirurgias programadas e destas $49,6 \%$ em regime de ambulatório. Constatou-se ainda a realização de 89608 procedimentos com apoio de anestesia fora do bloco operatório, 282944 consultas de anestesia e 112183 consultas de dor crónica. Identificaram-se 51380 analgesias de parto, que deverão ter correspondido a mais do que $85 \%$ dos partos ocorridos nos serviços de Obstetrícia das $\mathrm{IH}$ do SNS.

Foram identificados 1280 Anestesiologistas a trabalhar nas IH do SNS, o que corresponde a um rácio de 12,4 por 100000 habitantes. Se tivermos em conta os 262 Anestesiologistas a trabalhar exclusivamente no sector privado, esse rácio passa a ser de 15,1 por 100000 habitantes.

Apesar das limitações na metodologia utilizada para a identificação dos deficits nas $\mathrm{IH}$ analisadas, os diversos diretores dos serviços de Anestesiologia afirmam que, no seu todo, existem menos 541 Anestesiologistas que os necessários para as necessidades em cuidados anestésicos no SNS.

\section{AGRADECIMENTOS}

A Direção do Colégio de Anestesiologia gostaria de deixar uma palavra de agradecimento a todos os colaboradores que de forma directa ou indireta permitiram a recolha dos dados apresentados, nomeadamente a todos os diretores dos serviços de Anestesiologia das IH do SNS, diretores clínicos dos hospitais privados com atividade cirúrgica em Portugal, e mesmo Anestesiologistas a título individual que foram inexcedíveis no cumprimento dessa tarefa, contribuindo para o enriquecimento deste documento.

\section{REFERÊNCIAS}

1. Lemos P, Lima JF, Viana J, Assunção JP, Veiga J, Chedas M, et al. Censos Anestesiologia 2014 - Relatório Final. Rev Soc Port Anestesiol. 2015;24:41-52.

2. Pordata. Partos: total e em estabelecimentos de saúde, 2017. [consultado 2017 Set 11]. Disponível em: https://www.pordata.pt/Site/MicroPage. aspx?DatabaseName=Portugal $\&$ MicroName $=$ Partos+total+e+em+esta belecimentos+de+saúde\&MicroURL=152\&.

3. Portugal. Ministério da Saúde. Monitorização do Serviço Nacional de Saúde, 2016. [consultado 2017 Set 11]. Disponível em: http://benchmarking.acss.min-saude.pt.

4. Alran S, Sibony O, Oury JF, Luton D, Blot P. Differences in management and results in term-delivery in nine European referral hospitals: descrip-

tive study. Eur J Obstet Gynecol Reprod Biol. 2002;103:4-13.

5. Portugal. Ministério da Saúde. Rede Nacional de Especialidade Hospitalar e de Referenciação de Anestesiologia. [consultado 2017 Set 11]. Disponível em: https://www.sns.gov.pt/wp-content/uploads/2017/06/ RNEHR-Anestesiologia-Aprovado-Portal-SNS-19-06-2017.pdf.

6. WFSA Global Anesthesia Workforce Survey. Anesth Analg. 2017;125:981-90.

7. François Clergue. The challenges of anaesthesia for the next decade. Eur J Anaesthesiol. 2015;32:223-9.

8. Bartels K, Karhausen J, Clambey ET, Grenz A, Eltzschig HK. Peripoerative organ injury. Anesthesiology. 2013;119:1474-89. 